\title{
On an Iterative Method for Direction of Arrival Estimation using Multiple Frequencies
}

\author{
Fredrik Andersson and Marcus Carlsson \\ Department of Mathematics \\ Lund University, Sweden \\ fa@maths.lth.se, marcus.carlsson@math.lu.se
}

\author{
Jean-Yves Tourneret and Herwig Wendt \\ IRIT-ENSEEIHT, CNRS UMR 5505 \\ University of Toulouse, France \\ firstname.lastnamedirit.fr
}

\begin{abstract}
We develop a method for the estimation of the location of sources from measurements at multiple frequencies, including wideband measurements, recorded by a linear array of sensors. We employ interpolation matrices to address unequal sampling at different frequencies and make use of the Kronecker theorem to cast the nonlinear least squares problem associated with direction of arrival estimation into an optimization problem in the space of sequences generating Hankel matrices of fixed rank. We then obtain approximate solutions to this problem using the alternating direction method of multipliers. The resulting algorithm is simple and easy to implement. We provide numerical simulations that illustrate its excellent practical performance, significantly outperforming subspace-based methods both at low and high signal-to-noise ratio.
\end{abstract}

\section{INTRODUCTION}

The problem of estimating the location of sources emitting energy, or direction of arrival (DOA) estimation, from measurements registered by an array of passive sensors occurs in a large variety of applications of various fields, including telecommunications, radar, sonar, astrophysics and surveillance, to name but a few. Let $2 J+1$ identical receivers be located in a uniform linear array (ULA) with spacing $\Delta$, i.e. at positions $x_{j}=j \Delta, j=-J, \ldots, J$, let the $P$ sources be located sufficiently far away from the array so that the signals emitted by source $p, p=1, \ldots, P$, can be assumed to be plane waves at arrival at the array after travelling through a homogeneous medium. Furthermore, we assume $P$ to be known. Denote by $r_{p}(x, t)$ the signal from source $p$ recorded by the receiver at location $x$, and $\widehat{r}_{p}(x, \omega)=\int_{-\infty}^{\infty} r_{p}(x, t) e^{-2 \pi i t \omega} d t$ its Fourier transform with respect to time. From the plane wave assumption on the incident waves it follows that

$$
\widehat{r}_{p}(x, \omega)=e^{2 \pi i x \cos \left(\theta_{p}\right) \omega} \widehat{r}_{p}(0, \omega),
$$

a relation that holds for each frequency $\omega$.

Let $r(x, t)=\sum_{p=1}^{P} r_{p}(x, t)+n_{p}(x, t)$ denote the actual measurements and $\widehat{r}(x, \omega)$ their Fourier transforms, where $n_{p}(x, t)$ is additive noise (below, we will omit the noise term whenever appropriate). It then holds that for each fixed frequency $\omega$ the function $\widehat{r}(x, \omega)$ is a sum of $P$ exponentials,

$$
\begin{array}{r}
\widehat{r}(x, \omega)=\sum_{p=1}^{P} \widehat{r}_{p}(0, \omega) e^{2 \pi i x \cos \left(\theta_{p}\right) \omega}=\sum_{p=1}^{P} c_{p, \omega} e^{x \eta_{p}} \\
\eta_{p}=2 \pi i \cos \left(\theta_{p}\right) \omega \text { and } c_{p, \omega}=\widehat{r}_{p}(0, \omega) .
\end{array}
$$

For single fixed frequency $\omega=\omega_{c}$ (and approximately so for narrowband sources), the DOA estimation problem is therefore equivalent to the problem of estimating the parameters $\boldsymbol{\eta}=$ $\left(\eta_{1}, \cdots, \eta_{P}\right)^{T}$ that determine the position of $P$ spectral lines. Many of the classical spectral estimation techniques can be directly applied to this problem (cf., e.g., [1] for an overview), in particular high-resolution methods specifically designed for the estimation of line spectra, such as the popular subspace methods (e.g., MUSIC [2] and ESPRIT [3]), methods aiming at solving the nonlinear least squares problem associated with (2), cf. [4], as well as the Hankel matrix and alternating direction method of multipliers (ADMM) based formulation recently developed by the authors in [5].

The objective of the present contribution is to develop a novel high-resolution methodology for the estimation of the parameters $\boldsymbol{\eta}$, and thus obtain the directions $\boldsymbol{\theta}=$ $\left(\theta_{1}, \cdots, \theta_{P}\right)^{T}$, by jointly using information from multiple frequencies $\boldsymbol{\omega}=\left(\omega_{1}, \cdots, \omega_{M}\right)^{T}$, without any specific assumptions on the spectra of the sources. To this end, we will construct operators (matrices) from the measurements and enforce special structure on the operators that will allow us to make estimations of $\boldsymbol{\eta}$. More specifically, the proposed methodology relies on the following original key ingredients: First, we make use of Kronecker's theorem for complex symmetric matrices, which essentially states that the Hankel matrix that is generated by a vector of uniform samples $\left.\widehat{r}(j \Delta, \omega)\right|_{\omega=\omega_{c}}$ of a function $\left.\widehat{r}(x, \omega)\right|_{\omega=\omega_{c}}$ has $\operatorname{rank} P$ if and only if $\left.\widehat{r}(x, \omega)\right|_{\omega=\omega_{c}}$ coincides at the sample points with a function that is a linear combination of $P$ exponential functions. Second, for different values of $\omega_{m}$, the data entering the frequency estimation procedure are sampled at the stretched or contracted sample points $x_{j} \omega_{m}$ (compare (2)). In our formulation, we address this problem of unequally sampled data through the introduction of an auxiliary equally spaced grid and appropriate interpolation matrices. Finally, we reformulate the resulting minimization problem such that it can be effectively solved by ADMM [6], a robust and scalable iterative technique that has recently gained popularity due to its versatility while at the same time enjoying state-of-the-art performance. Although the optimization problem considered here can be non convex, numerical experiments indicate that the proposed ADMM procedure generically yields excellent estimates. The resulting algorithm is simple and easy to im- 
plement, and numerical results demonstrate the real practical benefits and excellent estimation performance as compared to classical subspace method based DOA estimation.

Alternative methods for high-resolution wideband direction of arrival methods include Coherent Signal Subspace Methods (CMS), cf. [7], [8] and approaches using extended ESPRIT [9].

\section{PROBLEM FORMUlation USING HANKEL MATRICES}

To simplify the presentation, we rescale the units so that $\Delta=1$ in the remainder of the paper. Recall that a Hankel matrix $A$ has constant values on the anti-diagonals, i.e., $\mathrm{A}(j, k)=$ $\mathrm{A}\left(j^{\prime}, k^{\prime}\right)$ if $j+k=j^{\prime}+k^{\prime}$. It can thus be generated element wise from a vector $\mathrm{f}$, i.e., $\mathrm{A}(j, k)=\mathrm{f}_{j+k-1}, 1 \leq j, k \leq N+1$. We denote the generation of the Hankel matrix $A$ from a vector $\mathrm{f}$ by $A=H \mathrm{f}$, and we denote the (adjoint) operation of summing a matrix A over its antidiagonals with $H^{*}$ A, i.e., $H^{*} \mathrm{~A}(n)=\sum_{l_{1}+l_{2}=n} \mathrm{~A}\left(l_{1}, l_{2}\right)$. Hankel matrices are complex symmetric and can be factorized as $A=\bar{U} \Sigma U^{*}$, where $\Sigma$ is a diagonal matrix with non-increasing diagonal elements $\sigma_{l}$, and the column vectors $\mathrm{u}_{l}$ are the so-called con-eigenvectors which are orthonormal and satisfy $\mathrm{Au}_{l}=\sigma_{l} \overline{\mathrm{u}_{l}}$.

It is well known that the Hankel matrix $A=H \mathrm{f}$ is of rank $P$ then, with the exception of degenerate cases, there exist $\left\{\eta_{p}\right\}_{p=1}^{P}$ and $\left\{c_{p}\right\}_{p=1}^{P}$ in $\mathbb{C}$ such that $f$ is sampled from the function

$$
f(x)=\sum_{p=1}^{P} c_{p} e^{\eta_{p} x},
$$

and the converse holds as we ${ }^{p} \overline{1}$. This is usually referred to as Kronecker's theorem, (see e.g. [10], [11], [12] for different versions). Moreover, if $\sigma_{l}=0$ then the polynomial generated from $\mathbf{u}_{l} Q_{\mathbf{u}_{l}}(\eta)=\sum_{k=0}^{N} \mathbf{u}_{l}(k+1) \eta^{k}$ has the property $Q_{\mathrm{u}_{l}}\left(\eta_{p}\right)=0$. This can be seen using (3):

$$
\begin{aligned}
0 & =\left(A \mathbf{u}_{l}\right)(j)=\sum_{k=1}^{N+1} A(j, k) \mathbf{u}_{l}(k)=\sum_{k=1}^{N+1} \sum_{p=1}^{P} c_{p} e^{\eta_{p}(j+k-1)} \mathbf{u}_{l}(k) \\
& =\sum_{p=1}^{P} c_{p} e^{\eta_{p} j} \sum_{k=1}^{N+1} e^{\eta_{p}(k-1)} \mathbf{u}_{l}(k)=\sum_{p=1}^{P} c_{p} e^{\eta_{p} j} Q_{\mathbf{u}_{l}}\left(\eta_{p}\right),
\end{aligned}
$$

which holds for $1 \leq j \leq N+1$ and as long as $P<N+1$, this is an overdetermined system for the values of $Q_{\mathrm{u}_{l}}\left(\eta_{p}\right)$, and hence $Q_{\mathrm{u}_{l}}\left(\eta_{p}\right)=0$ except for degenerate systems. A unit sampling distance was used here, yet the argument holds for arbitrary fixed distance. Consequently, it is possible to find the exponentials parameters $\eta_{p}$ for a function of the form (3) by sampling it, selecting a vector in the nullspace of the Hankel matrix generated from the samples, and determining the roots of the polynomial generated by this vector. The polynomial $Q_{\mathrm{u}_{l}}$ has $N$ roots, thus $N-P$ false roots are obtained by this procedure. The coefficients $c_{p}$ in (3) can be found by solving a linear (Vandermonde) system, and those associated with false roots equal zero in the absence of noise and are discarded.

Another important (generic) property of non-full rank Hankel matrices is that the con-eigenvectors $\mathrm{u}_{l}, 1 \leq l \leq P$ are also linear combination of exponentials as in (3), i.e.,

$$
\mathrm{u}_{l}(j)=\sum_{p=1}^{P} d_{l, p} e^{\eta_{p} j} .
$$

A requirement for two vectors (sampled functions) $f_{1}$ and $f_{2}$ to be generated by the same set of $P$ exponentials is thus that there exist orthonormal vectors $\mathrm{u}_{1}, \ldots, \mathrm{u}_{P}$ such that if $\mathrm{U}_{P}=\left[\mathrm{u}_{1}, \ldots, \mathrm{u}_{P}\right]$, then

$$
\overline{\mathcal{P}_{\mathrm{U}_{P}}} H \mathrm{f}_{1} \mathcal{P}_{\mathrm{U}_{P}}=H \mathrm{f}_{1}, \quad \overline{\mathcal{P}_{\mathrm{U}_{P}}} H \mathrm{f}_{2} \mathcal{P}_{\mathrm{U}_{P}}=H \mathrm{f}_{2},
$$

where $\mathcal{P}_{\mathrm{U}_{P}}=\mathrm{U}_{P} \mathrm{U}_{P}^{*}$ is the projection onto the span of $\mathrm{U}_{P}$.

The conditions above mean that $H \mathrm{f}_{1}$ and $H \mathrm{f}_{2}$ both have rank $P$ (because of the projection operator $\mathrm{U}_{P} \mathrm{U}_{P}^{*}$ ) and that both $f_{1}$ and $f_{2}$ are generated by the exponentials that generate $\mathrm{u}_{1}, \ldots, \mathrm{u}_{P}$. Clearly, the same argument can be used to compare if $\mathrm{f}_{1}, \ldots \mathrm{f}_{M}$ are generated by the same set of exponentials. In the noise free case, estimates of the exponential parameters can thus be immediately obtained for each fixed value of $\omega$.

In the presence of noise, one can consider rank $P$ approximations of Hankel matrices to estimate the exponential parameters. This can be formulated as an optimization problem,

$$
\begin{array}{ll}
\underset{\mathrm{g}}{\operatorname{minimize}} & \frac{1}{2}\|\mathrm{f}-\mathrm{g}\|_{2}^{2} \\
\text { subject to } & \operatorname{rank}(H \boldsymbol{g})=P,
\end{array}
$$

which is equivalent to the nonlinear least squares problem for estimating the parameters of the function (3) from samples with noise, cp. [5]. In [5] a method for obtaining an approximate solution to (5) is proposed for equally spaced data using the Alternate Direction Method of Multipliers (ADMM), and we follow a similar strategy here. To phrase the problem we need to make use of an auxiliary equally spaced grid and interpolation. If we can assume that the sampling at the equally spaced grid is sufficiently dense to represent the exponentials that we want to recover (i.e., the Nyquist sampling rate with proper adjustments for finite intervals), we can construct an interpolation matrix $K$ that maps samples between equally and unequally spaced grids $x^{\mathrm{eq}}$ and $x$, respectively.

$$
\begin{aligned}
& \text { Let } x^{\mathrm{eq}}(j)=\frac{j}{2 J},-J \leq j \leq J \text { and let } \\
& \qquad K(j, k)=\frac{\varphi\left(2 J\left(x^{\mathrm{eq}}(j)-x(k)\right)\right)}{\sum_{l} \varphi\left(2 J\left(x^{\mathrm{eq}}(l)-x(k)\right)\right)} .
\end{aligned}
$$

A commonly used $\varphi$ is the Lanczos function with $a$ lobes,

$$
\varphi(x)= \begin{cases}\operatorname{sinc}(x) \operatorname{sinc}(x / a) & \text { if } 0 \leq|x|<a, \\ 0 & \text { if }|x| \leq a .\end{cases}
$$

The counterpart of (5) for unequally spaced data is then

$$
\begin{array}{ll}
\underset{\mathrm{g}}{\operatorname{minimize}} & \frac{1}{2}\|\mathrm{f}-K \mathrm{~g}\|_{2}^{2} \\
\text { subject to } & \operatorname{rank}(H \boldsymbol{g})=P .
\end{array}
$$

\section{AN ADMM FORMULATION FOR DOA}

Assume that the measured data originate from $P$ sources and are contaminated by noise, and assume that there are measurements of $\hat{r}\left(j, \omega_{m}\right)$ available where $\omega_{m}<\omega_{m+1}$, $m=1, \ldots, M$, and $J$ is as in the previous section. Let $r(j, m)=\hat{r}\left(j, \omega_{m}\right)$, and let $r_{m}$ denote the vector obtained by fixing $m$. Also set $x_{m}(j)=j \omega_{m} /\left(2 J \omega_{M}\right)$. All the nodes $x_{m}(j)$ are then contained in the unit length interval $[-1 / 2,1 / 2]$. Let $N>J$, and let $x^{\mathrm{eq}}(n)=n /(2 N)$, $-N \leq n \leq N$, be an equally spaced lattice. Let $\mathrm{K}_{\mathrm{m}}$ be an interpolation matrix that interpolates from the equally spaced 
data $x^{\mathrm{eq}}$ to the (scaled) points $x_{m}$. By (1), the functions $x \mapsto \hat{r}\left(\frac{\omega_{M}}{\omega_{m}} x, \omega_{m}\right)$ are then linear combinations of the same $P$ exponential functions (plus noice) whose frequencies reveal $\boldsymbol{\theta}$. If $\mathrm{g}_{\mathrm{m}}$ denotes the above function sampled at $x^{\mathrm{eq}}$, we then have $\mathrm{K}_{\mathrm{m}} \mathrm{g}_{m}=\mathrm{r}_{\mathrm{m}}$.

Assume that the measured data originate from $P$ sources and are contaminated by noise. The DOA estimation problem using all the measurements $r\left(x(j), \omega_{m}\right),|j| \leq J, m=$ $1, \ldots, M$, can then be formulated as

$\underset{\mathrm{g}}{\operatorname{minimize}} \sum_{m=1}^{M}\left\|\mathrm{r}_{\mathrm{m}}-\mathrm{K}_{\mathrm{m}} \mathrm{g}_{m}\right\|_{2}^{2}$

such that $\exists \eta_{p}, 1 \leq p \leq P: \mathrm{g}_{\mathrm{m}}(j)=\sum_{p} d_{m, p} e^{\eta_{p} j}, \forall|j| \leq J$.

The matrices $\mathrm{K}_{\mathrm{m}}$ interpolate the function represented by the vector $g_{m}$ on the equally spaced grid $x^{\text {eq }}$ to the equally spaced grid $x_{m}$. The objective function thus describes the discrepancy between the interpolation between functions $g_{m}$ sampled at $x^{\text {eq }}$ and the measured data $r_{m}$.

We now reformulate (7) such that it can be addressed using ADMM. Let

$\mathcal{R}_{P}\left(\left\{\mathrm{~A}_{\mathrm{m}}\right\}\right)= \begin{cases}\infty & \nexists U_{P}=\left[\mathrm{u}_{1}, \ldots, \mathrm{u}_{P}\right]: \mathrm{A}_{\mathrm{m}}=\overline{\mathcal{P}_{\mathrm{U}_{P}}} \mathrm{~A}_{\mathrm{m}} \mathcal{P}_{\mathrm{U}_{P}}, \forall m, \\ 0 & \text { otherwise. }\end{cases}$

The role of $\mathcal{R}_{P}$ is to enforce finite ranks on the matrices $\mathrm{A}_{m}$, which in combination with the Hankel constraint means that the generating vectors $\mathrm{g}_{m}$ are linear combination of $P$ exponentials. Instead of using this approach, one could replace this type of finite rank condition with a nuclear norm based counterpart. This has the advantage of giving rise to convex optimization problems, yet also has disadvantages. This field has received considerable attention over the recent years and a thorough discussion is beyond the scope of this paper. We remark that it is straightforward to adapt the methodology proposed here to nuclear norm type of penalties.

To arrive at an algorithm, we reformulate (7) as

$$
\underset{\mathrm{g}}{\operatorname{minimize}} \mathcal{R}_{P}\left(\left\{\mathrm{~A}_{\mathrm{m}}\right\}\right)+\sum_{m=1}^{M}\left\|\mathrm{r}_{\mathrm{m}}-\mathrm{K}_{\mathrm{m}} \mathrm{g}_{m}\right\|_{2}^{2}
$$

subject to $\mathrm{A}_{\mathrm{m}}(k, l)=\mathrm{g}_{\mathrm{m}}(k+l), \quad-N \leq k, l \leq N$

The rank constraint of (7) is now contained in $\mathcal{R}_{P}\left(\left\{A_{m}\right\}\right)$ in the objective function while the condition $\mathrm{A}_{\mathrm{m}}(k, l)=$ $\mathrm{r}_{\mathrm{m}}(k+l)$ forces the matrices $\mathrm{A}_{\mathrm{m}}$ to have Hankel structure, i.e. to equal $H \mathrm{~g}_{\mathrm{m}}$. Let us introduce the notation $\langle A, B\rangle=$ $\operatorname{Re}\left(\sum_{k, l=-N}^{N} A(k, l) \overline{B(k, l)}\right)$, as a scalar product on complex valued matrices.For the ADMM formulation we define (the augmented Lagrangian)

$$
\begin{aligned}
& L\left(\left\{\mathrm{~A}_{\mathrm{m}}\right\},\left\{\mathrm{g}_{\mathrm{m}}\right\},\left\{\Lambda_{\mathrm{m}}\right\}\right)=\mathcal{R}_{P}\left(\left\{\mathrm{~A}_{\mathrm{m}}\right\}\right)+\sum_{m=1}^{M}\left\|\mathrm{r}_{\mathrm{m}}-\mathrm{K}_{\mathrm{m}} \mathrm{g}_{m}\right\|_{2}^{2} \\
& +\sum_{m=1}^{M}\left\langle\Lambda_{\mathrm{m}}, \mathrm{A}_{\mathrm{m}}-H \mathrm{~g}_{\mathrm{m}}\right\rangle_{\mathbb{R}}+\rho\left\|\mathrm{A}_{\mathrm{m}}-H \mathrm{~g}_{\mathrm{m}}\right\|_{F}^{2},
\end{aligned}
$$

where $\|A\|_{F}^{2}=\langle A, A\rangle_{\mathbb{R}}$ denotes the Frobenius norm.

The ADMM steps for (8) then read, for $1 \leq m \leq M$

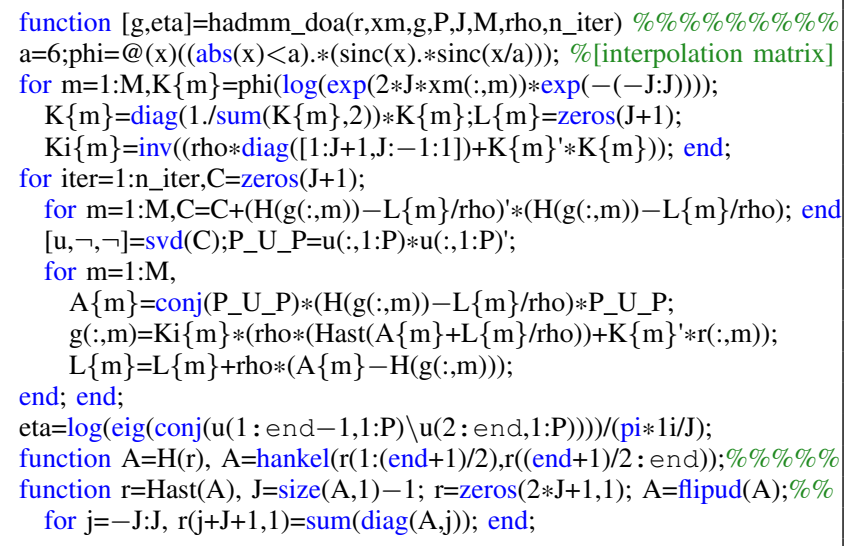

$\mathrm{A}\{\mathrm{m}\}=$ conj(P_U_P $) *(\mathrm{H}(\mathrm{g}(:, \mathrm{m}))-\mathrm{L}\{\mathrm{m}\} /$ rho $) * \mathrm{P} \_$U_P $\mathrm{g}(:, \mathrm{m})=\mathrm{Ki}\{\mathrm{m}\} *\left(\operatorname{rho} *(\operatorname{Hast}(\mathrm{A}\{\mathrm{m}\}+\mathrm{L}\{\mathrm{m}\} / \mathrm{rho}))+\mathrm{K}\{\mathrm{m}\}^{\prime} * \mathrm{r}(:, \mathrm{m})\right)$; $\mathrm{L}\{\mathrm{m}\}=\mathrm{L}\{\mathrm{m}\}+\mathrm{rho} *(\mathrm{~A}\{\mathrm{~m}\}-\mathrm{H}(\mathrm{g}(:, \mathrm{m})))$;

TABLE I: Main function (in MATLAB syntax) for approximation using exponentials and DOA estimation for $P$ sources
1) $\left\{\mathrm{A}_{\mathrm{m}}{ }^{k+1}\right\}=\operatorname{argmin} L\left(\left\{\mathrm{~A}_{\mathrm{m}}\right\},\left\{\mathrm{g}_{\mathrm{m}}{ }^{k}\right\},\left\{\Lambda_{\mathrm{m}}{ }^{k}\right\}\right)$,
$\left\{A_{m}\right\}$
2) $\left\{g_{\mathrm{m}}{ }^{k+1}\right\}=\operatorname{argmin} L\left(\left\{\mathrm{~A}_{\mathrm{m}}{ }^{k+1}\right\},\left\{\mathrm{g}_{\mathrm{m}}\right\},\left\{\Lambda_{\mathrm{m}}{ }^{k}\right\}\right)$,
3) $\Lambda_{\mathrm{m}}{ }^{k+1}=\Lambda_{\mathrm{m}}{ }^{k}+\rho\left(\mathrm{A}_{\mathrm{m}}{ }^{k+1}-H \mathrm{~g}_{\mathrm{m}}{ }^{k+1}\right)$.

For the first minimization step, by dropping the terms that are independent of $A_{m}$ and by completion of squares, we have

$$
\mathrm{A}_{\mathrm{m}}{ }^{k+1}=\underset{\mathrm{A}_{\mathrm{m}}}{\operatorname{argmin}} \mathcal{R}_{P}\left(\left\{\mathrm{~A}_{\mathrm{m}}\right\}\right)+\rho \sum_{m}\left\|\mathrm{~A}_{\mathrm{m}}-H \mathrm{~g}_{\mathrm{m}}+\Lambda_{\mathrm{m}} / \rho\right\|_{2}^{2} .
$$

The solution can be obtained as follows. We define

$$
\mathrm{C}_{\mathrm{m}}=\sum_{m}\left(H \mathrm{~g}_{\mathrm{m}}-\Lambda_{\mathrm{m}} / \rho\right)^{*}\left(H \mathrm{~g}_{\mathrm{m}}-\Lambda_{\mathrm{m}} / \rho\right) .
$$

Since $C_{m}$ is a Hermitian matrix it can be diagonalized as $\mathrm{C}_{\mathrm{m}}=\mathrm{U} \Sigma \mathrm{U}^{*}$, where $\Sigma$ is a diagonal matrix containing the eigenvalues of $C_{m}$ in descending order, and where the corresponding eigenvectors are the orthonormal columns of $U$. Let $U_{P}=\left[u_{1}, \ldots, u_{P}\right]$. The matrices $A_{m}$ that solve (9) are then given by a projection of the complex symmetric matrices $\mathrm{A}_{\mathrm{m}}{ }^{k+1}-\mathrm{Hg}_{\mathrm{m}}{ }^{k+1}$ to Takagi factorizations using the span of the $P$ eigenvectors of $\mathrm{C}$ corresponding to the $P$ largest eigenvalues of C, i.e., $A_{m}=\overline{\mathcal{P}_{\mathrm{U}_{P}}}\left(H \mathrm{~g}_{\mathrm{m}}-\Lambda_{\mathrm{m}} / \rho\right) \mathcal{P}_{\mathrm{U}_{P}}$.

Next, we turn our focus to the second ADMM step. In a similar way as for the first step, we drop terms independent on $g_{m}$ and complete squares. In this case, the vectors $g_{m}$ can be analyzed individually for each fixed $m$,

$$
\mathrm{g}_{m}^{k+1}=\underset{\mathbf{g}_{m}}{\operatorname{argmin}}\left\|\mathrm{K}_{\mathrm{m}} \mathrm{g}_{m}-\mathrm{r}_{\mathrm{m}}\right\|^{2}+\rho\left\|H \mathrm{~g}_{\mathrm{m}}-\mathrm{A}_{m}^{k+1}-\Lambda_{m}^{k} / \rho\right\|^{2} .
$$

Then it is easy to see (following the same idea as in [5]) that (10) is minimized by solving

$$
\left(\rho \operatorname{diag}(\mathrm{w})+\mathrm{K}_{\mathrm{m}}{ }^{*} \mathrm{~K}_{\mathrm{m}}\right) \mathrm{g}_{\mathrm{m}}=\rho H^{*}\left(\mathrm{~A}_{\mathrm{m}}+\Lambda_{\mathrm{m}} / \rho\right)+\mathrm{K}_{\mathrm{m}}{ }^{*} r_{m},
$$

where $\mathrm{w}(n)=N+1-n,-N \leq n \leq N$ is a triangle weight.

Having solved the two minimization steps in the ADMM procedure allows us to state a short and simple algorithm for the direction of arrival estimation problem:

\section{RESUlts}

We illustrate the performance of the proposed methods by means of numerical simulations with $P=8$ sources, 31 


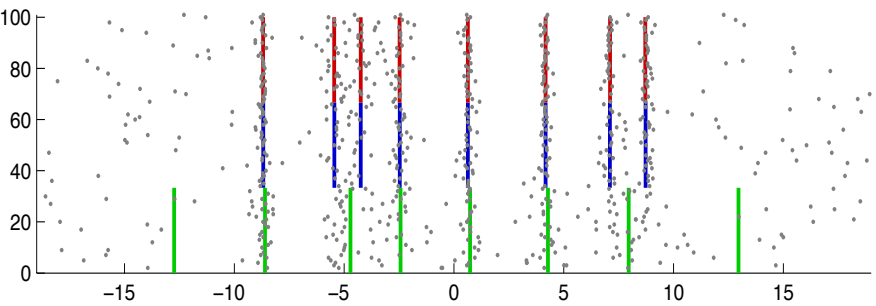

Fig. 1: DOA estimation for $P=8$ sources and $M=100$ frequencies at SNR level of $10 \mathrm{~dB}$. The gray circles indicate estimates for each individual frequency using ESPRIT, green lines the medians over frequencies of ordered estimates, red lines the true locations and blue lines the estimates obtained by the proposed method.

\begin{tabular}{|c|ccccccc|}
\hline SNR (dB) & -6 & -3 & 0 & 3 & 6 & 10 & 30 \\
\hline Method & & & & & & \\
\hline Proposed method & 0.9490 & 0.2524 & 0.0340 & 0.0206 & 0.0145 & 0.0089 & 0.0011 \\
ESPRIT median & 5.5291 & 5.0863 & 4.5198 & 4.0377 & 3.5199 & 2.3760 & 0.0077 \\
ESPRIT average & 5.8845 & 5.4908 & 4.9904 & 4.3871 & 3.7559 & 2.9479 & 0.7995 \\
ESPRIT individual & 7.3210 & 6.9264 & 6.4591 & 5.9231 & 5.3405 & 4.6308 & 2.4268 \\
\hline
\end{tabular}

TABLE II: Standard deviation in estimated directions using the metric describes in Section IV.

receivers $(J=16)$ and using $M=100$ frequencies. With respect to the normalized units $(\Delta=1)$, an affine frequency range with $\omega_{1}=0.5$ and $\omega_{M}=1$ is used. A fixed set of parameters $\eta_{p}$ is generated by a quasi-random generator on the interval $-\frac{2 J}{3} \leq \eta_{p} \leq \frac{2 J}{3}$ and is indicated by red lines in Figure 1. The measured data $\widehat{r}(x, \omega)$ are then generated using $\eta_{p}$ along with a pseudo-random coefficients $c_{p, \omega}$ for the individual frequencies as in (2), with white Gaussian noise added to each measurement vector $r_{m}$ for varying signal-tonoise ratio (SNR, with respect to total power of all vectors $\left.r_{m}\right)$. Results obtained with the proposed method using all frequencies $\boldsymbol{\omega}$ are compared to those obtained using ESPRIT for each frequency $\omega_{m}$ individually. These latter estimates are also used to obtain initial values of $g_{m}$ by extrapolation. Furthermore, we extract overall ESPRIT based estimates by first ordering the estimates at each frequency individually and then computing the median and the average over all frequencies for each of the $P$ estimates.

In Figure 1, results obtained for one single noise realization with SNR of $10 \mathrm{~dB}$ are plotted. The positions of the correct values of $\eta_{p}$ are indicated by red lines, and those of the real part of the values estimated with the proposed method by blue lines. ESPRIT estimates for each individual frequency $\omega_{m}$ are displayed by gray dots, green lines indicate the median for frequencies of each of the 8 ordered estimates. Clearly, the estimates obtained by the proposed method are substantially better than the estimates obtained by ESPRIT, the latter suffering from very poor estimation quality at frequencies $\omega_{m}$ with little signal power, and in particular so at low frequencies.

Next, we study the estimation performance using 100 noise realizations, respectively, for various SNR levels. We sort the 8 detected sources and compare the estimates with the true values by measuring standard deviations. For the ESPRIT version, we in three ways: for each $\omega_{m}$ individually; for the mean over the sorted estimates over all $\omega_{m}$; and for the median of the sorted estimates over all $\omega_{m}$.

The results are summarized in Table II. We see that the proposed method consistently outperforms ESPRIT based estimation both for low and high SNR, and that it still provides reasonably good results even for low SNR.

\section{CONCLUSions}

We have proposed a novel high-resolution method for direction of arrival estimation based on multiple frequency or wideband measurements. The method makes use of the Kronecker theorem for implicitly imposing the model equations for each considered frequency through rank penalization of the Hankel matrices generated by the corresponding approximating sequences. The resulting optimization problem is then resolved using ADMM. Although the problem is nonconvex (and hence inherits the problem of local minima), numerical simulations demonstrate that the method yields excellent practical performance. In particular, it significantly outperforms ESPRIT based estimation, both at low and high SNR levels, and enables to obtain practically usable estimates even at very low SNR. Furthermore, the proposed method yields an algorithm that is simple and easy to implement.

\section{REFERENCES}

[1] P. Stoica and R. Moses, Spectral analysis of Signals, Prentice-Hall, 2005.

[2] R. Schmidt, "Multiple emitter location and signal parameter estimation," IEEE Trans. on Antennas and Propagation, vol. 34, no. 3, pp. 276 280, 1986.

[3] R. Roy and T. Kailath, "ESPRIT-estimation of signal parameters via rotational invariance techniques," IEEE Trans. on Acoustics, Speech and Signal Process., vol. 37, no. 7, pp. 984-995, 1989.

[4] Y. Bresler and A. Macovski, "Exact maximum likelihood parameter estimation of superimposed exponential signals in noise," IEEE Trans. Acoustic, Speech and Signal Process., vol. 34, no. 5, pp. 1081-1089, 1986.

[5] F. Andersson, M. Carlsson, J.-Y. Tourneret, and H. Wendt, "Frequency estimation based on Hankel matrices and the alternating direction method of multipliers," in Proceedings of EUSIPCO, Marrakesh, 2013, to appear. preprint arXiv:1306.2907.

[6] S. Boyd, N. Parikh, E. Chu, B. Peleato, and J. Eckstein, "Distributed optimization and statistical learning via the alternating direction method of multipliers," Foundations and Trends ${ }^{\circledR}$ in Machine Learning, vol. 3, no. 1, pp. 1-122, 2011.

[7] H Wang and M Kaveh, "Coherent signal-subspace processing for the detection and estimation of angles of arrival of multiple wide-band sources," IEEE Trans. Acoustics, Speech and Signal Process.,, vol. 33, no. 4 , pp. 823-831, 1985.

[8] Hsiensen Hung and Mostafa Kaveh, "Focussing matrices for coherent signal-subspace processing," Acoustics, Speech and Signal Processing, IEEE Transactions on, vol. 36, no. 8, pp. 1272-1281, 1988.

[9] Björn Ottersten and Thomas Kailath, "Direction-of-arrival estimation for wide-band signals using the esprit algorithm," Acoustics, Speech and Signal Processing, IEEE Transactions on, vol. 38, no. 2, pp. $317-$ 327, 1990.

[10] L. Kronecker, "Zur Theorie der Elimination einer Variablen aus zwei algebraischen Gleichungen," Monatsber. K. Preuss. Akad. Wiss., pp. 535-600, 1881 .

[11] R.L. Ellis and D.C. Lay, "Factorization of finite rank Hankel and Toeplitz matrices," Linear algebra and its applications, vol. 173, pp. 19-38, 1992.

[12] R. Rochberg, "Toeplitz and Hankel operators on the Paley-Wiener space," Integ. Eq. and Op. Th., vol. 10, 1987. 